\title{
ÉTICA E INVESTIGACIÓN
}

\author{
Salomón Zavala1,2,a, Julio Alfaro-Mantilla1,3,b
}

\begin{abstract}
RESUMEN
Los autores hacen una evaluación crítica de los aspectos éticos de la investigación en base a su experiencia como docentes universitarios y como miembros de Comités de Ética en Investigación. Invitan a la discusión de temas que consideran polémicos. Comienzan mencionando el rol regulador de las Normas Éticas Internaciones y Locales y de los Comités de Ética en Investigación. Comentan la postura de bioeticistas sudamericanos respecto del llamado "doble estándar" ético y sobre la liberalización del uso del placebo. Critican el perjuicio que ocasiona a los pacientes de bajos recursos el sistema de patentes así como la falta de interés en el desarrollo de nuevos medicamentos para tratar enfermedades raras o propias de países pobres, y la excesiva extensión de los Consentimientos Informados. Terminan opinando acerca de la distribución de los ensayos clínicos entre los investigadores, los problemas que afectan a los Comités de Ética en Investigación y sobre algunos contenidos del Reglamento de Ensayos Clínicos del Instituto Nacional de Salud.
\end{abstract}

Palabras clave: Bioética; Ética médica; Derechos humanos (fuente: Decs BIREME).

\section{ETHICS AND INVESTIGATION}

\begin{abstract}
The authors make a critical evaluation of the ethical aspects of research based on their experience as university teachers and members of Ethics Committees. They invite to the discussion on topics that they consider polemic. They begin by mentioning the regulatory role of the International and Local Ethical Norms and of the Ethics Committees. They comment on the position of South American bioethicists regarding the so-called ethical "double standard" and on the liberalization of the use of the placebo. They criticize the damage that the system of patents causes on low- resources patients, as well as the lack of interest in the development of new medications to treat neglected diseases or those diseases which are only prevalent in poor countries, and the excessive length of the Informed Consents. They finish giving their opinion about the distribution of the clinical trials among the researchers, the problems that affect the Ethics Committees and some contents of the Regulation of Clinical Trials of the National Institute of Health.
\end{abstract}

Key words: Bioethics; Ethics medical; Human rights (source: MeSH NLM).

Vinculados desde hace un buen número de años a la docencia universitaria, a la investigación como ejecutores o asesores y a la vigilancia ética a través de comités de ética de investigación, nos permitimos realizar una serie de reflexiones que, si motivan discusiones, controversias, desacuerdos o nuevos enfoques, habrán cumplido sus objetivos.

\section{INVESTIGACIÓN EN SERES HUMANOS}

Desde que Albert Neisser, el investigador alemán que descubrió el gonococo, fue juzgado y sancionado en 1898 por haber inyectado suero de enfermos sifilíticos a otros pacientes y a prostitutas, sin su consentimiento ni conocimiento, ello como parte de investigaciones orientadas a la prevención de la sífilis, se han conocido múltiples casos de faltas graves a la ética en investigación, algunas de ellas con ribetes de escándalo tales como el conocido estudio de Tuskegee (1) o el más reciente caso de Guatemala ${ }^{(2)}$. En este último, médicos de los Servicios de Salud de los Estados Unidos infectaron con sífilis y gonorrea a presos, soldados y pacientes de hospitales psiquiátricos, sin su consentimiento, con el fin de estudiar el efecto de la penicilina en el tratamiento y la prevención de esta enfermedad. La inminente publicación de ese estudio ha provocado que el Gobierno de Estados Unidos pida perdón a Guatemala, país en el que se realizó el experimento entre los años 1946 y 1948.

\footnotetext{
Instituto de Ética en Salud, Universidad Nacional Mayor de San Marcos. Lima, Perú.

2 Comité de Ética de Investigación, Asociación PRISMA. Lima, Perú.

3 Comité de Ética en Investigación del Hospital Edgardo Rebagliati Martins. Lima, Perú.

a Médico Internista; ${ }^{b}$ Médico Neurólogo
} 
El desarrollo de las normas éticas y jurídicas universales, entre ellas el Código de Nuremberg, la Declaración de Helsinki, el Informe Belmont y la Declaración Universal sobre Bioética y Derechos Humanos de la UNESCO aspiran a limitar y controlar los abusos en la utilización de las ciencias y las tecnologías asociadas a las biotecnologías, limitar las tendencias invasivas del mercado, e impulsar y proteger los derechos fundamentales de las personas.

Al respecto, los principios éticos generales que rigen la investigación en seres humanos, de acuerdo con la Council for International Organizations of Medical Sciences (CIOMS), son tres: a) El respeto absoluto por las personas, fundamentalmente referida a la autonomía del sujeto quien debe decidir libremente su participación, y aun si ha decidido participar, pueda decidir su retiro del estudio si así lo cree conveniente; y la protección de las personas vulnerables, es decir, con autonomía limitada, a quienes se les debe ofrecer las seguridades del caso; b) Beneficencia, referida a la obligación ética de maximizar los beneficios y disminuir la probabilidad de daño, es decir que una investigación no sea nunca maleficente; y, c) Justicia, particularmente la que considera la distribución equitativa de los riesgos y beneficios del estudio entre todos los participantes ${ }^{(3)}$.

Con la misma finalidad se establecieron los comités de ética en investigación (CEI), definidos como "Un grupo multidisciplinario e independiente de profesionales de la salud, así como de otros campos del conocimiento, y miembros de la comunidad, que tiene por objetivo contribuir a salvaguardar la dignidad, los derechos y el bienestar de los participantes actuales y potenciales de la investigación, asegurando que los beneficios e inconvenientes de la investigación sean distribuidos equitativamente entre los grupos y clases de la sociedad, y resguardando la relevancia y corrección científica del protocolo de investigación que se somete a su consideración" (4). En Perú, el Ministerio de Salud a través del Instituto Nacional de Salud (INS) es el ente regulador de los ensayos clínicos.

\section{PROBLEMAS BIOÉTICOS ACTUALES EN INVESTIGACIÓN CIENTÍFICA}

\section{VÍNCULO CON LA INDUSTRIA FARMACÉUTICA}

La necesidad de acortar la duración de los estudios de investigación sobre medicamentos, aunada al alto nivel científico alcanzado por los profesionales de muchos países del tercer mundo, el Perú entre ellos, ha permitido el desarrollo de ensayos clínicos multicéntricos que actualmente se llevan a cabo exitosamente, sin objeciones éticas y con ventajas para pacientes, instituciones e investigadores; aunque hay quienes sostienen que la extensión de los ensayos clínicos a los países en desarrollo se debe a que en estos las regulaciones son más laxas y el costo de la investigación es menor ${ }^{(5)}$.

A la industria farmacéutica se le atribuyen, en algunos casos, maniobras destinadas a incrementar sus ganancias extendiendo la vigencia de sus patentes y acelerando la conclusión de investigaciones para conseguir la aprobación de medicamentos que, después se comprueba, no tienen la eficacia atribuida y producen efectos adversos que obligan a suspender su comercialización ${ }^{(6)}$.

En recientes eventos realizados en nuestro país, los expertos en Bioética, Juan Carlos Tealdi, y Volnei Garrafa (7-10) llamaron la atención y denunciaron la política de los Institutos de Salud de los Estados Unidos y de la U.S. Food and Drug Administration (FDA), acusándolos de crear el llamado "doble estándar", una ética para los países ricos y otra para los países pobres, refiriéndose a investigaciones realizadas en algunos países en desarrollo en pacientes con $\mathrm{VIH}$, y de haber impulsado la liberalización del uso del placebo.

El sistema de patentes, aceptado sin objeciones en empresas de negocios, en el caso de la industria farmacéutica impide que millones de personas de bajos recursos puedan beneficiarse de nuevos medicamentos para aliviar sus males, sin poder disponer de los llamados genéricos, de mucho menor costo. Esta situación no tiene coherencia con frases tales como la que inicia la Declaración de Helsinski: La ciencia debe estar al servicio de la humanidad, por lo que de algún modo tendrá que cambiar en el futuro. ¿Tiene sentido que el sacrificio de los pacientes que participan en los ensayos clínicos y el esfuerzo de los miembros de los comités de ética, que actúan por altruismo, sirva primariamente para incrementar los ingresos de los accionistas de las empresas farmacéuticas?

\section{PRIORIDADES DE INVESTIGACIÓN}

¿Quién establece las prioridades sobre el tipo de drogas o en que área de acción se debe investigar? Si se sigue la mentalidad de un inversionista, es lógico y legítimo que la prioridad esté dada por los probables réditos que la droga, si es exitosa, rendirá. Esto dependerá, entre otras variables, de la frecuencia y tiempo de uso. Una droga que tenga alta frecuencia de uso y se administre por tiempo prolongado, como puede ser un fármaco para tratar la hipertensión arterial, enfermedad que como sabemos, salvo rarísimas excepciones, se 
controla pero no se cura, prometerá mayor rédito económico. Esto ha dado lugar a que las enfermedades crónicas no trasmisibles sean las que generen mayor interés para la búsqueda de fármacos en investigación.

Medicamentos huérfanos y enfermedades raras. Se llama así a medicamentos para enfermedades raras, cuya poca frecuencia en la población general hace que no sean de interés para la industria farmacéutica. El bajo número de pacientes a los que se dirigirá el fármaco, representa una dificultad, primero a nivel de desarrollo (por ser poco numeroso el grupo de pacientes en los que se podrá estudiar la eficacia del fármaco), y también por la baja rentabilidad que conllevará la inversión. Por tanto, las autoridades sanitarias asumen que es preciso proteger su desarrollo e incentivarlo con fondos públicos, ya que estos pacientes, aunque escasos, tienen el mismo derecho a la salud que otros con enfermedades más comunes. Estos medicamentos que necesitan una especial atención para desarrollarse se denominan "medicamentos huérfanos", porque "han de cuidarse como si fuesen niños sin padres" (11).

Se afirma que hay unas 5000 enfermedades raras, generalmente debidas a alteraciones genéticas para cuyo tratamiento no existen fármacos. Esto dio lugar en los EE.UU. en 1983, a la llamada Orphan Drug Act ${ }^{(12)}$, para tratar de corregir esta deficiencia. Así, por ejemplo, se planteó una reducción de impuestos del $50 \%$, para la inversión en este tipo de investigación. Se sabe también que Japón, Australia y la Unión Europea están elaborando un reglamento para estos incentivos.

Sin embargo, hay otras enfermedades, nada raras o infrecuentes, para las cuales no hay estudios farmacológicos pese a su urgente necesidad de atención. Preguntémonos, por ejemplo, ¿cuánto tiempo hace que no tenemos un nuevo fármaco para el tratamiento de la tuberculosis?

Como este vacío no puede quedar sin cobertura de manera indefinida tenemos más preguntas: ¿Quién debe cubrirlo, los gobiernos, las universidades, o se debe estimular o crear mecanismos como el planteado por el Orphan Drug Act?

Extensión del consentimiento informado. Cuántas veces hemos escuchado a miembros de los $\mathrm{CEI}$ comentar lo difícil que se les hace revisar consentimientos informados de los protocolos de ensayos clínicos, de 25 a 30 páginas de extensión, y preguntarse si el participante realmente lee y entiende todo su contenido o calcular ¿cuánto tiempo le tomará al investigador explicarles todo el contenido a cada uno de ellos?

Nos parece que la extensión responde, en gran parte, a la necesidad de cubrir los posibles conflictos o reclamos que pudieran surgir, por ello estimamos que son los asesores legales los que tienen importante participación en su diseño ¿Se podrá encontrar un "punto medio" de equilibrio?

Tabla 1. Modelo de consentimiento informado para investigación clínica.

Investigador responsable

Título del protocolo

Título del acta del consentimiento

Se me ha solicitado participar en un estudio de investigación que está estudiando ........(describir brevemente en términos corrientes los procedimientos en los cuales el sujeto está de acuerdo)

YO ENTIENDO QUE:

A) Los posibles riesgos de este procedimiento incluyen... ( se confecciona una lista de los riesgos conocidos o de los efectos colaterales; si no hay tales riesgos, esto debe establecerse). Los tratamientos alternativos incluyen... (confeccionar una lista de tratamientos alternativos y describir brevemente las ventajas y desventajas de cada uno: si no existieran, esto debe establecerse)

B) Los posibles beneficios que tendré en este estudio son:... (enumerarlos; si no hay beneficios, esto debe establecerse).

C) Cualquier pregunta que yo quisiera hacer en relación a mi participación en este estudio deberá ser contestada por... (deberá hacerse una lista de nombres y cargos de personas que deberán responder a las preguntas).

D) Yo podré retirarme de este estudio en cualquier momento sin dar razones ni tampoco sin que esto me perjudique.

E) Los resultados de este estudio pueden ser publicados, pero mi nombre o identidad no será revelada y mis datos clínicos y experimentales permanecerán en forma confidencial, a menos que mi identidad sea solicitada por ley.

F) Mi consentimiento está dado voluntariamente sin que haya sido forzado u obligado.

G) En el caso que sea dañado físicamente como resultado del estudio, la atención y el tratamiento médico serán proporcionados en esta institución.

Fecha 
Un ejemplo que puede ser útil para la comprensión del lector, es el que encontramos en el libro "Ética clínica" de la Universidad de Chile, página 163d, de C. Quintana y M. Lavados ${ }^{(13)}$ que citamos en la Tabla 1.

Una alternativa podría ser agregar un resumen del documento de consentimiento informado en el que se exprese con toda claridad: que es una investigación, que como toda investigación implica riesgos aunque sean mínimos, que su participación es voluntaria y en qué consiste su participación. Esto es lo que los participantes debieran necesariamente comprender y recordar. Lo demás del documento lo podrían consultar, leer y releer tanto como quieran.

Número de protocolos por investigador. ¿Existe un número límite de protocolos que un mismo investigador puede tener en un momento dado?

Aquí existen varias situaciones: a) El investigador tiene varios protocolos, pero solo uno o dos pacientes por cada uno; o b) El investigador tiene varios protocolos y un buen número de participantes por cada uno de ellos.

Desde luego que, mientras más estudios estén a cargo de un investigador, mayor será el riesgo de que cometa algún descuido o error. Sin embargo, un investigador experimentado con muchos estudios en su haber genera más confianza que un novato con un solo estudio. Por lo tanto el número de estudios no puede ser el único criterio. Las insinuaciones de "democratizar la investigación", es decir repartir ¿equitativamente? los ensayos clínicos entre los médicos interesados, aparte de ser un absurdo, hace pensar que las críticas que se hacen a los investigadores que llevan muchos estudios tiene otras motivaciones. Controlar que los investigadores no descuiden su trabajo asistencial es tarea de sus jefes y autoridades y ese control debe ser naturalmente muy estricto.

¿Por qué un investigador es requerido para hacer varios estudios a la vez? Esto generalmente se debe a razones como el prestigio personal, confianza ganada en investigaciones previas, contar con un buen número de investigadores secundarios y con una buena organización de apoyo y auxiliares, trabajar en un servicio con alta demanda de los pacientes requeridos, etc.

Al margen de las razones o explicaciones mencionadas, creemos que quien debe establecer el límite de protocolos a realizar en un mismo periodo, es el propio investigador, basado en su sentido de responsabilidad y ética profesional. Los CEI están facultados para poner un límite considerando cada caso particular.

Comités de ética en investigación. En la actualidad, según la relación que publica el INS en su página web ${ }^{(14)}$, en el Perú existen $30 \mathrm{CEI}$ aprobados. De estos, doce pertenecen a hospitales públicos, ocho a institutos nacionales como: Instituto de Salud del Niño, Instituto Nacional de Enfermedades Neoplásicas, Instituto Nacional de Oftalmología, Instituto Nacional de Rehabilitación, Instituto de Ciencias Neurológicas. Instituto Materno Perinatal e Instituto de Salud Mental; seis a organismos no gubernamentales (ONG): Instituto de Investigación Nutricional, PRISMA, IMPACTA, VIA LIBRE, Piura y el NAMRU-6; y 4 pertenecen a universidades: Nacional Mayor de San Marcos (UNMSM), Peruana Cayetano Heredia, San Martin de Porres y el Instituto D. A. Carrión de la UNMSM.

Compensación a los miembros de los CEI. Un problema que rara vez se toca respecto a los $\mathrm{CEI}$ es ¿qué tipo de compensación deben recibir sus miembros por su participación? En algunos hospitales, a los miembros del CEI se les asigna horas laborales que apenas alcanzan a cubrir su participación en las sesiones, mas no se les reconoce el tiempo que dedican a la lectura de los protocolos, la búsqueda de información relacionada y la elaboración de su informe.

Estipendio a los miembros de los comités. Nuestra propuesta es que la autoridad regulatoria establezca una modalidad de compensación que sea razonable y uniforme. Los recursos existen puesto que según el Reglamento de Ensayos Clínicos del INS ${ }^{(15)}$ los comités "Pueden cobrar una tarifa para cubrir sus costos operativos", aunque, por ahora, y por lo menos en algunos Comités, tenemos información directa de que el dinero recaudado no está llegando a su destino. La modalidad de dieta por asistencia a las sesiones tendría la ventaja de contribuir a asegurar el quórum.

En los CEI privados tenemos información que en solo dos de ellos se asigna a los miembros un estipendio, más simbólico que real, por el tiempo que dura su intervención. Tal vez por concepto de lucro cesante, siendo el presidente quien recibe una mayor cantidad en proporción a su dedicación.

Si tomamos en cuenta el tiempo dedicado, los conocimientos requeridos y la responsabilidad implícita, este tema debería discutirse en cada comité para ver cómo se resuelve, sin pensar equivocadamente que no es ético recibir una compensación ya que esta corresponde al tiempo dedicado a la tarea y no por la naturaleza de su opinión.

Independencia de los comités. Una de las características que diferencia a los CEI de los demás comités hospitalarios es que aquellos requieren un alto grado de independencia para poder cumplir 
con la obligación de proteger a los sujetos de investigación. Sin duda, por falta de conocimiento, algunos directivos, cometiendo falta grave, han cambiado abruptamente a los miembros de su comité por no ser de su agrado.

El Reglamento de Ensayos Clínicos. En el Reglamento de Ensayos Clínicos del Instituto Nacional de Salud (16) se extrañan algunos ítems que regulen las obligaciones de las instituciones de investigación tales como el apoyo a los comités, el respeto a su independencia, la implementación de los centros de investigación, el uso de los recursos del overhead y su vinculación con el INS, etc.

Overhead. Las instituciones de investigación cobran a los patrocinadores de ensayos clínicos un monto equivalente a un porcentaje del presupuesto total del estudio, mal llamado overhead (costos indirectos) por concepto de utilización del campo clínico. Uno de los tantos aciertos del Reglamento de Ensayos Clínicos es la Décima Segunda Disposición Complementaria: Fondo Intangible para Fines de Investigación (17) por la cual se dispone que Los fondos provenientes del contrato entre la institución del sector público y el patrocinador serán considerados como fondo intangible para fines de investigación, actividad que no suele ser prioritaria ¿Se estará cumpliendo?

Tesis universitarias. Respecto a las tesis universitarias, los CEI tropiezan con un problema que dificulta su actividad: las tesis, en su gran mayoría, tienen fallas metodológicas que no han sido corregidas por sus asesores o instituciones. Si una investigación no es metodológicamente correcta no puede ser ética y, por ende, no debe ser autorizada.

Supervisión de ensayos clínicos. EI INS exige a los CEI supervisar el desarrollo de los ensayos clínicos. Robert Levine ha argumentado contra la supervisión de rutina por parte de los CEI diciendo lo siguiente: Puede afectar la atmósfera de confianza entre el comité y los investigadores, los procedimientos de monitoreo son indebida o inconvenientemente costosos y convertirían al comité en una fuerza fiscalizadora, cuando su mayor valor es educacional ${ }^{(18)}$.

Ninguno de los documentos regulatorios internacionales conocidos ni de publicaciones relacionadas menciona supervisión como una actividad propia de los CEI (19). Hablan de continuing review cuya traducción literal es "revisión continuada", a través de los informes que los investigadores envían por obligación con una periodicidad que los comités establecen para cada estudio, por lo menos una vez al año.
Consideramos que el mayor valor de un CEI es su composición multiprofesional y variopinta que le permite deliberar considerando todos los puntos de vista y tomar las mejores decisiones. Sus miembros actúan motivados por altruismo, una facultad que se les puede agradecer, pero no exigir sin límites. Cuando es preciso, ejercen su autoridad, que les permite revisar documentos, historias clínicas, visitar los sites (instituciones donde se realiza el ensayo clínico), solicitar informes, entrevistar a pacientes e investigadores, etc., y así cumplir con su obligación a cabalidad. Por otro lado, los gerentes o directores de los hospitales están presionados para cumplir con las metas y para aumentar la productividad de sus instituciones y en ese contexto no tienen disposición para sacrificar horas médicas por algo que no les interesa directamente.

Parece que a nadie le importara establecer el costo de las actividades que realizan los miembros de un comité, considerando el valor de su tiempo en razón de su nivel profesional, a pesar de que sin ese conocimiento no se puede planificar adecuadamente. El resultado podría ser sorprendente.

\section{REDCEI}

Los CEI en nuestro medio están agrupados, por propia iniciativa, en una Institución denominada REDCEI que no tiene precedentes en otros países. La finalidad de la REDCEI es establecer un vínculo entre los comités de ética en investigación que la conforman para compartir conocimientos, experiencias, información, opiniones y facilidades para el mejor desempeño de sus funciones. Esta finalidad se ha cumplido exitosamente gracias principalmente al empeño y compromiso de sus coordinadoras, Roxana Lescano y Estela Quiroz. La REDCEI en el Perú ha sabido mantener su independencia respecto de la industria farmacéutica y de la propia autoridad regulatoria.

\section{REFERENCIAS BIBLIOGRÁFICAS}

1. Brandt AM. Racism and research: the case of the Tuskegee study. En: Emanuel EJ, Crouch RA, Arras JD, Moreno JD, Grady C, editors. Ethical and Regulatory Aspects of Clinical Research. 1ra ed. Baltimore: The Johns Hopkins University Press; 2003. p. 20-4.

2. Stein R. U.S. apologizes for newly revealed syphilis experiments done in Guatemala. Washington Post. USA; Octubre 1, 2010.

3. Council for International Organizations of Medical Sciences (CIOMS), Word Health Organization (WHO). International ethical guidelines for biomedical research involving human subjects. Geneva: CIOMS; 2002. 
4. Vidal S. Comités de ética de la investigación. En: Tealdi JC (director). Diccionario Latinoamericano de bioética. Bogotá: UNESCO-Universidad Nacional de Colombia; 2008.

5. Donohue JM, Cevasco M. A decade of direct-to-consumer advertising of prescription drugs. N Engl J Med. 2007; 357(7):673-81.

6. Topol EJ. The lost decade of nesiritide. N Engl J Med. 2011;365(1):81-2.

7. Garrafa V. Fortalecimiento de los Comités de Ética en Investigación en el Perú. Simposio Internacional del INS. Lima, Perú. Instituto Nacional de Salud. 2010.

8. Tealdi JC. Seminario de Bioética y Derechos Humanos. Observatorio de Bioética y Derechos Humanos. Lima, Perú. Pontificia Universidad Católica del Perú. 2010.

9. Tealdi JC. Investigación en Salud. En: UNESCO; Red Latinoamericana y del Caribe de Bioética. Seminario de Bioética y Derechos Humanos. Bogotá, Colombia. Universidad Nacional de Colombia. 2008.

10. Garrafa V, Lorenzo C. Helsinki 2008. Una Visión Crítica Latinoamericana. Bioética \& Debat (Instituto Borja). 2009;18:5-13.

11. Sommi RW Jr. The story of orphan drugs. Current Health. 1990;16(8):14.

12. U.S. Food and Drug Administration, Department of Health and Human Services. The Orphan Drug Act. Silver Spring, MD: FDA; c2011.

13. Quintana C, Lavados M. Ética clínica. Santiago: Universidad de Chile; 1993.

14. Instituto Nacional de Salud del Perú. Reglamento de ensayos clínicos. Lima: Instituto Nacional de Salud; 2011.
15. Instituto Nacional de Salud del Perú. De los Comités de Ética en Investigación. Capítulo VII. En: Instituto Nacional de Salud. Reglamento de Ensayos Clínicos. 1d ed. Lima: Instituto Nacional de Salud; 2010. p. 51-1.

16. Instituto Nacional de Salud del Perú. De las Personas y Entidades que participan en la Ejecución de los Ensayos clínicos. Título IV. En: Instituto Nacional de Salud. Reglamento de Ensayos Clínicos. 1d ed. Lima: Instituto Nacional de Salud; 2010. p. 42-54.

17. Instituto Nacional de Salud del Perú. Disposiciones Complementarias. En: Instituto Nacional de Salud. Reglamento de Ensayos Clínicos. 1d ed. Lima: Instituto Nacional de Salud; 2010. p. 82-2.

18. Weijer $\mathrm{CH}$, Shapiro SH, Fuks A, Glass KC, Skrutkowska M. Monitoring clinical research: an obligation unfulfilled. En: Emanuel EJ, Crouch RA, Arras JD, Moreno JD, Grady $\mathrm{C}$, editors. Ethical and Regulatory Aspects of Clinical Research. 1ra ed. Baltimore: The Johns Hopkins University Press; 2003. p. 425-5.

19. Amdur RJ, Bankert EA. Continuing review of research. En: Institutional Review Board. Member Handbook. 1a ed. Massachusett: Jones and Bertlett publishers; 2007. p. 53-5.

Correspondencia: Julio Alfaro-Mantilla

Dirección: Avenida Pérez Araníbar 1032, Magdalena del Mar.

Lima, 17

Teléfono: (51) 264-0516/264-2896

Correo electrónico: jucam@amauta.rcp.net.pe

\section{Consulte las ediciones anteriores de la} Revista Peruana de Medicina Experimental y Salud Pública en www.scielo.org.pe

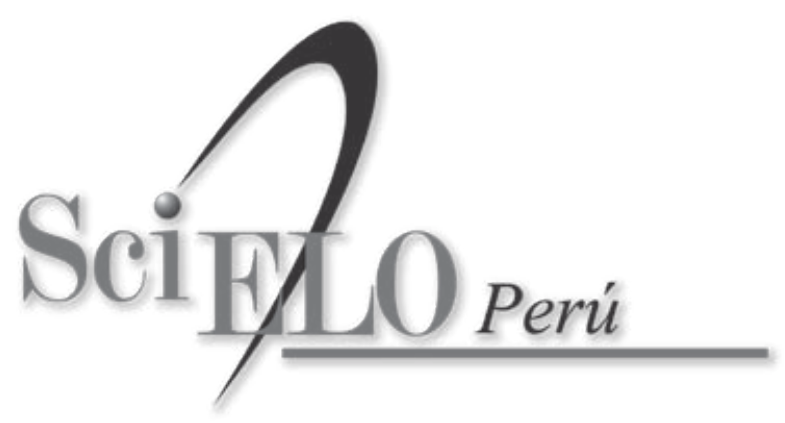

